\title{
Educação Alimentar e Nutricional como prática de intervenção: reflexão e possibilidades de fortalecimento
}

\section{I ${ }^{1}$ Ana Maria Cervato-Mancuso, ${ }^{2}$ Kellem Regina Rosendo Vincha, ${ }^{3}$ Débora Aparecida Santiago I}

Resumo: Introdução: As intervençôes nutricionais são configuradas, historicamente, por distribuição de alimentos e ampliação de conhecimento, permanecendo nas práticas atuais. Para ampliar o campo criou-se, em 2012, o Marco de Referência de Educação Alimentar e Nutricional. Objetivo: Analisar a produção científica brasileira sobre avaliação de intervenções de Educação Alimentar e Nutricional antes da criação do marco. Metodologia: Pesquisa sistemática com inclusão de artigos publicados entre 2000-2012. Após leitura crítica, foram feitas divisão e seleção dos estudos, classificando-os por região, contexto e cenário. Resultados: Selecionaram-se 28 artigos, divididos em três categorias: características institucionais e do estudo; contexto da intervenção e atores sociais envolvidos; e estruturação e base teórica. Verificou-se que os nutricionistas, assim como o tema da nutrição, estão mais presentes no contexto da saúde. No ambiente escolar, no território e no ambiente de trabalho, a promoção da saúde é abordada por equipes multiprofissionais. Os objetivos e as avaliações das intervenções relacionam-se, predominantemente, com indicadores numéricos, mas os estudos do território se aproximam dos referenciais do marco ao se apropriarem de indicadores qualitativos. Observou-se descrição sucinta das bases teórico-metodológicas, limitando a reprodução dos estudos. Consideraçóes: Aponta-se a necessidade de realizar mais estudos de intervenção e de aperfeiçoá-los mediante a utilização do marco como referência.

> Palavras-chave: Educação Alimentar e Nutricional; estudo de intervenção; revisão.

\footnotetext{
1 Faculdade de Saúde Pública Universidade de São Paulo. São Paulo-SP, Brasil (cervato@ usp.br).

2 Faculdade de Saúde Pública, Universidade de São Paulo. São Paulo-SP, Brasil (kvincha@ yahoo.com.br).

${ }^{3}$ Faculdade de Saúde Pública, Universidade de São Paulo. São Paulo-SP, Brasil (debora. santiago@hotmail.com).
}

Recebido em: 17/10/2014 Aprovado em: 25/10/2015 


\section{Introdução}

Ao longo do tempo, as sociedades adquirem normas e hábitos de consumo alimentar, aos quais incorporam um processo de experimentação social que, por tentativa e erro, propicia o desenvolvimento de um saber nutricional (CASTRO; COIMBRA, 1985). Diante disso, considerar que os hábitos alimentares de determinada população precisam ser alterados é um grande desafio para as políticas públicas e para a educação em nutrição, dado que esses envolvem relações entre pessoas e comportamentos humanos (BOOG, 2013).

No Brasil, as primeiras intervenções governamentais no campo da alimentação, motivadas pelas preocupações da ciência de nutrição, ocorreram na década de 1940. Nesse período, a educação alimentar sofreu influências de ordem política e ideológica, centralizando-se no binômio "alimentação e educação". Assim, os programas procuraram associar a distribuição de alimentos a ações de caráter educativo, com o intuito de ampliar os conhecimentos sobre alimentação racional e econômica da população (CASTRO; PELIANO, 1985).

Já nas décadas de 70 e 80, o binômio deu lugar à relação "alimentação e renda", consolidando a postulação de que pobres sabiam comer, mas lhes faltava renda para comprar alimentos. A partir dessa concepção, os programas de distribuição de alimentos passaram a ser vistos como instrumentos de redistribuição de renda. A preocupação com proteínas e com a educação foi substituída pela fome, má distribuição de renda e preocupação com o pequeno produtor (CASTRO; PELIANO, 1985). Nessa época, as estratégias de intervenção tomaram caminhos diametralmente opostos, a partir da identificação dos fatores determinantes. Se desnutrição resultava da ignorância, a cura seria a criação de programas educativos e a introdução de novos alimentos. Se a desnutrição resultava da pobreza, a cura seria a transformação do modelo de desenvolvimento econômico (CASTRO; PELIANO, 1985).

Documentos da Organização Pan-Americana da Saúde, Organização Mundial da Saúde e Organização das Nações Unidas para Alimentação e Agricultura, nas décadas de 40, 50 e 70, apontavam que a educação nutricional era uma missão de todos. Esses, organizados em conteúdos e estratégias de forma vertical e internacional, eram disseminados para diferentes trabalhadores, incluindo agrônomos, economistas, médicos e trabalhadores sanitários em geral, 
que recebiam treinamento e orientação sobre métodos de ensino para atuarem junto à população (BOOG, 1997).

$\mathrm{Na}$ década de 90, os estudiosos da área foram surpreendidos com a emergência de um novo fenômeno - a obesidade como problema de saúde pública - que rompeu a concepção que a má alimentação relacionava-se estritamente à falta de renda (BOOG, 2011). Consequentemente, houve uma reformulação dos documentos, referindo que a educação nutricional inclui, além do processo de ensino e aprendizagem, técnicas de planejamento e avaliação. Afinal, enquanto as intervenções preocupavam-se com as questôes econômicas, as mudanças dos hábitos alimentares da população configuravam um descompasso na atuação profissional (BOOG, 1997).

Uma vez iniciadas as ações de alimentação e nutrição, as dificuldades dos profissionais na abordagem para as mudanças dos hábitos foram emergindo, tendo em vista a complexidade que rege o comportamento alimentar. Tais dificuldades estavam escoradas no fato de os cursos de saúde abordarem a nutrição com foco nos aspectos dos nutrientes e das fontes alimentares (BOOG, 2008).

$\mathrm{Na}$ década seguinte, houve uma crescente importância da temática nos documentos que elaboraram as políticas públicas de saúde e nas ações governamentais, especialmente as desenvolvidas pelos Ministérios do Desenvolvimento Social e de Combate à Fome, da Saúde e da Educação (SANTOS, 2012). Igualmente, ampliou-se também a configuração das ações de alimentação e nutrição, no sentido de uma educação emancipatória, regida pelas concepções da promoção da saúde (BOOG, 2011). Entretanto, mesmo com a crescente valorização da educação nutricional nos últimos anos, tem-se o consenso da dificuldade de desenvolver intervenções em decorrência da baixa existência de referenciais teóricos, metodológicos e operacionais (SANTOS, 2012).

Com o intuito de enfatizar a teoria e de respaldar a prática, em 2012, a Coordenação Geral de Educação Alimentar e Nutricional do Ministério do Desenvolvimento Social e Combate à Fome realizou atividades para construir, de forma coletiva, o documento denominado Marco de Referência de Educação Alimentar e Nutricional para as Políticas Públicas (BRASIL, 2012), que adotou o termo "Educação Alimentar e Nutricional" (EAN), definindo-o como:

[...] campo de conhecimento e de prática contínua e permanente, transdisciplinar e multiprofissional que visa promover a prática autônoma e voluntária de hábitos 
alimentares saudáveis. A prática da EAN deve fazer uso de abordagens e recursos educacionais problematizadores e ativos que favoreçam o diálogo junto a indivíduos e grupos populacionais [...] (BRASIL, 2012, p. 23).

Como observado, a EAN estabeleceu-se como um campo de prática profissional cujas ações podem e devem envolver nutricionistas e outros profissionais, permitindo, ainda, que estes tenham acesso a programas de formação e educação permanente. Nesse contexto, destacam-se: a formulação e ampliação do acesso a métodos de ensino específicos para a formação em EAN; a ampliação e a valorização de atividades de integração teórico-práticas; a articulação do ensino de EAN com os campos de conhecimento em ciências humanas (Sociologia, Antropologia, Ética e Filosofia); a ampliação da utilização de referências teóricas da área de Pedagogia e Educação; e o investimento na educação permanente dos docentes responsáveis pela disciplina.

Ao ler as referências bibliográficas do documento (BRASIL, 2012), verificase que muitos pesquisadores e profissionais brasileiros da área de Alimentação e Nutrição participaram e contribuíram na sua sustentação teórica e científica. Entretanto, a inexistência de uma produção científica que aborde os aspectos educativos reforça a ideia de que ainda não estão claros os procedimentos metodológicos necessários para o ensino e a pesquisa em EAN.

O objetivo do presente estudo foi analisar a produção científica brasileira sobre avaliação de intervenções de Educação Alimentar e Nutricional no período de 2000 até a criação do Marco de Referência de Educação Alimentar e Nutricional, em 2012. Assim, propôs-se identificar, de maneira reflexiva, as publicaçôes de autores brasileiros no formato de artigos e categorizá-las, de acordo com a afiliação institucional dos autores, tipologia e métodos do estudo, contexto da intervenção, indicadores de avaliação e resultados encontrados.

\section{Metodologia}

Como critérios de inclusão, foram selecionados artigos publicados no período de 2000-2012 tendo, pelo menos, um autor brasileiro. A pesquisa bibliográfica foi realizada por meio da base de dados do Scientific Eletronic Library Online (Scielo), utilizando os descritores: educação nutricional, educação alimentar, aconselhamento nutricional e letramento nutricional - no formulário base - 
e a combinação de aconselhamento alimentar com hábitos alimentares e com práticas alimentares e o grupo de palavras "programa, educação e nutrição" - no formulário livre. Os artigos repetidos foram descartados (figura 1).

Após o levantamento, fez-se uma avaliação dos artigos por meio da crítica de títulos e resumos, resultando na divisão de quatro grupos: estudos de avaliação de intervenção $(\mathrm{n}=28)$; estudos sobre métodos de intervenção, tendo a intervenção educativa ou o conteúdo como objetivo $(n=43)$; estudos de reflexão teórica ou histórica, revisão bibliográfica e análise documental $(n=55)$; e estudos de diagnóstico da situação institucional, incluindo determinantes da situação nutricional, aspectos ligados à qualificação da equipe de trabalho e tratamento utilizado $(n=224)$.

Figura 1. Etapas de identificação e seleção de artigos para a elaboração da pesquisa bibliográfica sobre intervenções de Educação Alimentar e Nutricional, 2000 a 2012

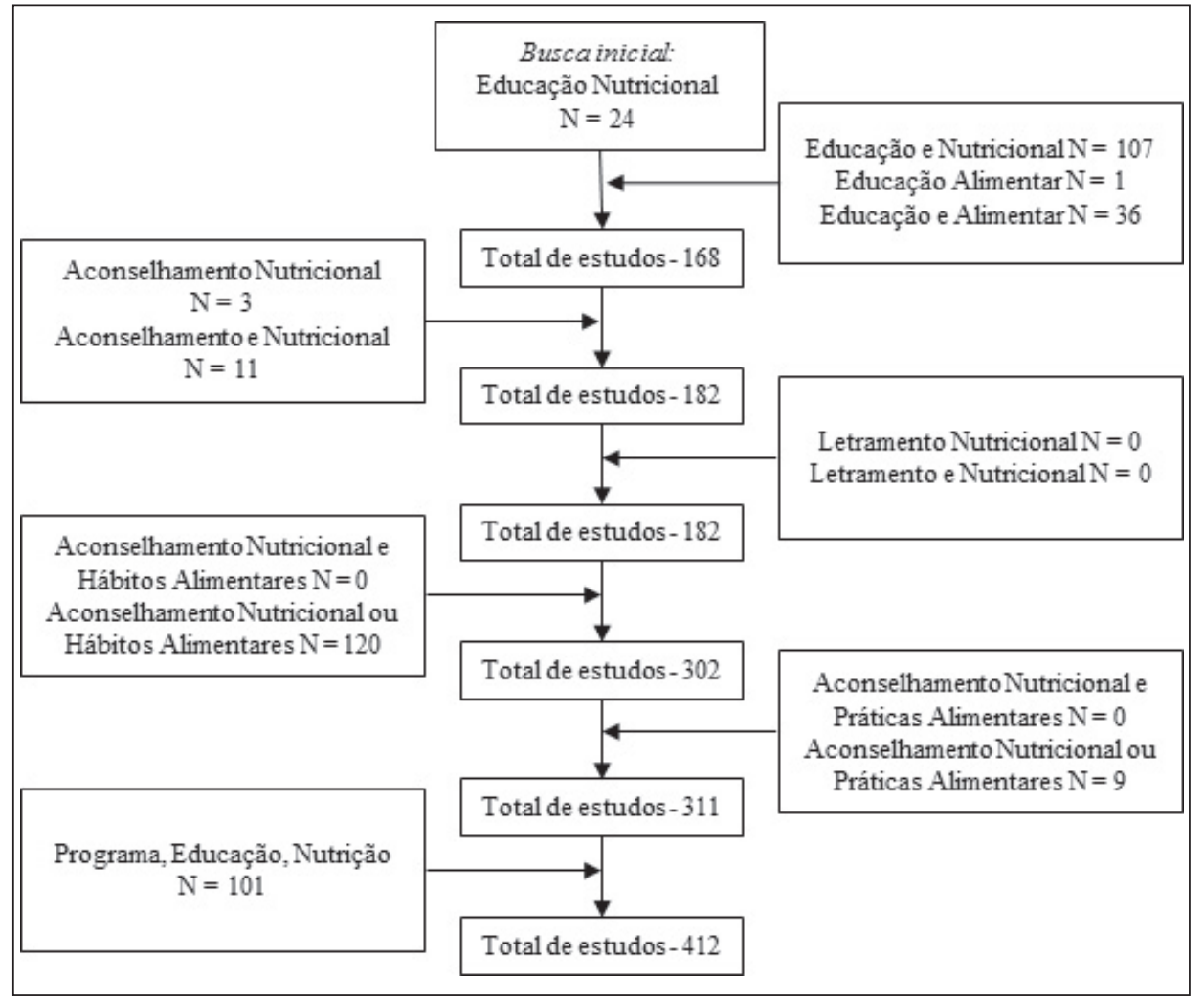

Fonte: elaboração própria. 
Durante a avaliação dos textos encontrados na pesquisa, alguns foram excluídos $(n=62)$, devido à inexistência da relação com os processos educativos - apesar da identificação inicial - ou por envolverem animais ou experimento com alimentos. Para o presente manuscrito, foram selecionados os estudos que realizaram avaliação de intervenção, aqui classificados segundo região, contexto e cenário da intervenção educativa.

\section{Resultados}

Foram selecionados 28 artigos, sendo que uns mencionam a intervenção e outros detalham o processo ou programa educativo. As semelhanças identificadas quanto à intervenção da EAN possibilitaram sua classificação em categorias de análise, apresentadas nos quadros que se seguem.

\section{Características institucionais e do estudo}

Dos 28 artigos, 12 tiveram suas análises conduzidas no estado de São Paulo (LESSA; DEVINCENZI; SIGULEM, 2003; DAVANÇO; TADDEI; GAGLIANONE, 2004; ADDE; RODRIGUES; CARDOSO, 2004; CERVATO et al., 2005; ALMEIDA-PITITTO et al., 2012; ATTUX et al., 2011; BOOG, 2010; BUENO et al., 2011; FAZIO et al., 2011; GAGLIANONE et al., 2006; JAIME et al., 2007; SILVA et al., 2006), especialmente por universidades públicas, com predomínio da Universidade de São Paulo e da Universidade Federal de São Paulo.

A principal característica presente nos estudos conduzidos em São Paulo é a avaliação pré e pós-intervenção com a presença de grupo controle. Vale destacar, porém, o artigo de Bueno et al. (2011), que se diferenciou dos demais ao avaliar uma intervenção por meio de um estudo retrospectivo, utilizando dados de prontuários de pacientes com obesidade, em tratamento.

Os estudos realizados em outros estados (ALENCAR; BARROS JÚNIOR; CARVALHO, 2008; ARAUJO; FIGUEIREDO; D'AVILA, 2010; BAENA et al., 2011; BOEHS et al., 2005; BUSNELLO et al., 2011; CARVALHO; FONSECA; PEDROSA, 2004; CRUZ; MORIMOTO, 2004; FELIPPE et al., 2011; FERNANDES et al., 2009; FUJIMORI et al., 2008; GABRIEL; SANTOS; VASCONCELOS, 2008; LIMA et al., 2011; MENDONÇA; LOPES, 2012; SANTOS et al., 2005, 2002; VARGAS et al., 2011) foram conduzidos por duas unidades da Pontifícia Universidade Católica (PUC) (Rio Grande do Sul e Paraná) e, principalmente, por universidades públicas federais. 
Além desses, encontrou-se um estudo (FELIPPE et al., 2011) cujo autor principal não possuía vínculo institucional, mas avaliava, em clínica particular, um programa de reeducação alimentar.

Além dos estudos de intervenção tipo pré e pós, com e sem grupo controle, também foram observadas, em outros estados, pesquisas de abordagem qualitativa, como o estudo etnográfico presente no artigo de Santos et al. (2002), o estudo descritivo dialético realizado por Alencar, Barros Júnior e Carvalho (2008), que analisou os aportes sócio-políticos em intervenção com idosos, e o estudo de narrativas de Lima et al. (2011).

\section{Contexto da intervenção e atores sociais envolvidos}

As atividades e os programas analisados nos estudos selecionados foram implementados em quatro espaços (tabela 1): ambiente escolar, serviço de saúde, território e ambiente de trabalho.

Tabela 1. Contexto da intervenção, conteúdos e atores envolvidos. São Paulo, 2013

\begin{tabular}{|c|c|c|c|c|c|}
\hline $1^{\circ}$ autor & Ano & Espaço & População & Conteúdo & Responsável \\
\hline Adde & 2004 & Saúde & Crianças & Nutrição & Nutricionista e médico \\
\hline Alencar & 2008 & Território & Idosos & $\begin{array}{l}\text { Nutrição e aspectos } \\
\text { sócio-políticos }\end{array}$ & Não especifica \\
\hline $\begin{array}{l}\text { Almeida- } \\
\text { Pititto }\end{array}$ & 2012 & Território & Adultos & $\begin{array}{c}\text { Nutrição e atividade } \\
\text { física }\end{array}$ & $\begin{array}{l}\text { Nutricionista e } \\
\text { educador físico }\end{array}$ \\
\hline Araujo & 2010 & Saúde & $\begin{array}{l}\text { Pacientes em } \\
\text { hemodiálise }\end{array}$ & $\begin{array}{l}\text { Nutrição e } \\
\text { metabolismo }\end{array}$ & Não especifica \\
\hline Attux & 2011 & Saúde & $\begin{array}{c}\text { Pacientes com } \\
\text { transtornos mentais }\end{array}$ & $\begin{array}{l}\text { Nutrição, atividade } \\
\text { física e autoestima }\end{array}$ & $\begin{array}{c}\text { Equipe } \\
\text { multiprofissional }\end{array}$ \\
\hline Baena & 2011 & Trabalho & $\begin{array}{l}\text { Trabalhadores e } \\
\text { familiares }\end{array}$ & $\begin{array}{c}\text { Nutrição e atividade } \\
\text { física }\end{array}$ & Nutricionista \\
\hline Boehs & 2005 & Território & Famílias & $\begin{array}{l}\text { Nutrição e } \\
\text { psicossocial }\end{array}$ & $\begin{array}{l}\text { Equipe } \\
\text { multiprofissional, com } \\
\text { residente de nutrição }\end{array}$ \\
\hline Boog & 2010 & Escolar & Alunos & Nutriçãoo & Nutricionista \\
\hline Bueno & 2011 & Território & Adultos obesos & $\begin{array}{c}\text { Nutrição e atividade } \\
\text { física }\end{array}$ & $\begin{array}{c}\text { Equipe } \\
\text { multiprofissional }\end{array}$ \\
\hline
\end{tabular}




\begin{tabular}{|c|c|c|c|c|c|}
\hline $1^{\circ}$ autor & Ano & Espaço & População & Conteúdo & Responsável \\
\hline Busnello & 2011 & Saúde & $\begin{array}{l}\text { Pacientes com } \\
\text { síndrome } \\
\text { metabólica }\end{array}$ & Nutrição & Não especifica \\
\hline Carvalho & 2004 & Território & Idosos & $\begin{array}{l}\text { Osteoporose, nutrição } \\
\text { e atividade física }\end{array}$ & $\begin{array}{c}\text { Equipe } \\
\text { multidisciplinar }\end{array}$ \\
\hline Cervato & 2005 & Território & Adultos e idosos & Nutrição & Nutricionista \\
\hline Cruz & 2004 & Saúde & Pacientes obesos & Nutrição & Nutricionista \\
\hline Davanço & 2004 & Escolar & Professores & $\begin{array}{c}\text { Nutrição e atividade } \\
\text { física }\end{array}$ & $\begin{array}{c}\text { Equipe } \\
\text { multidisciplinar }\end{array}$ \\
\hline Fazio & 2011 & Saúde & Gestantes & Nutrição & Nutricionista \\
\hline Felippe & 2011 & Saúde & Pacientes obesos & Nutrição & $\begin{array}{c}\text { Equipe } \\
\text { multiprofissional }\end{array}$ \\
\hline Fernandes & 2009 & Escolar & Alunos & Nutrição & Não especifica \\
\hline Fujimori & 2008 & Escolar & Alunos & Nutriçãoo & Não especifica \\
\hline Gabriel & 2008 & Escolar & Alunos & Nutriçãoo & Não especifica \\
\hline Gaglianone & 2006 & Escolar & Professores e alunos & $\begin{array}{c}\text { Nutrição e atividade } \\
\text { física }\end{array}$ & $\begin{array}{l}\text { Equipe } \\
\text { multiprofissional }\end{array}$ \\
\hline Jaime & 2007 & Território & Família & Nutriçãoo & Não especifica \\
\hline Lessa & 2003 & Território & Crianças & Saúde e nutrição & Nutricionista \\
\hline Lima & 2011 & Saúde & $\begin{array}{c}\text { Pacientes portadores } \\
\text { do vírus hepatite } \mathrm{C}\end{array}$ & Nutrição & Nutricionista \\
\hline Mendonça & 2012 & Território & Adultos e idosos & $\begin{array}{c}\text { Nutrição e atividade } \\
\text { física }\end{array}$ & Estudante de nutrição \\
\hline Santos & 2005 & Território & Líderes & Saúde e nutrição & Não especifica \\
\hline Santos & 2002 & Território & $\begin{array}{l}\text { Médicos, mães e } \\
\text { crianças }\end{array}$ & $\begin{array}{c}\text { Nutrição e } \\
\text { amamentação }\end{array}$ & Não especifica \\
\hline Silva & 2006 & Saúde & $\begin{array}{l}\text { Pacientes } \\
\text { hipertensos e } \\
\text { diabéticos }\end{array}$ & Saúde & $\begin{array}{c}\text { Equipe } \\
\text { multiprofissional }\end{array}$ \\
\hline Vargas & 2011 & Escolar & Alunos & $\begin{array}{c}\text { Nutrição e atividade } \\
\text { física }\end{array}$ & Não especifica \\
\hline
\end{tabular}

Fonte: elaboração própria. 
Foram encontradas sete pesquisas realizadas em ambiente escolar, das quais cinco fizeram intervenção com os alunos, uma com os professores e uma com ambos. Observou-se que o papel da nutrição foi preponderante em todos esses estudos, sendo que em três o tema abordado incluía a atividade física. Contudo, os responsáveis pela intervenção educativa pouco foram explicitados, de modo que apenas três artigos contemplaram esse item apresentando o nutricionista como coordenador da ação ou a equipe multiprofissional composta por médico, educador físico e nutricionista.

Dentre os estudos realizados em serviços de saúde, seis aconteceram em ambulatório hospitalar (ADDE; RODRIGUES; CARDOSO, 2004; ARAUJO; FIGUEIREDO; D'AVILA, 2010; ATTUX et al., 2011; BUSNELLO et al., 2011; FAZIO et al., 2011; LIMA et al., 2011), 2 em clínicas particulares (CRUZ; MORIMOTO, 2004; FELIPPE et al., 2011) e um em Unidade Básica de Saúde (UBS) (SILVA et al., 2006), de modo que a intervenção teve o nutricionista como responsável único em três estudos, compartilhado com o médico em um e com a equipe multiprofissional de saúde em três. A responsabilidade da intervenção não foi explicitada em dois estudos.

Quanto ao tema central das ações, a maioria abordou a nutrição direcionada a indivíduos portadores de doenças. Diferentemente dessa configuração de temática exclusiva, Attux et al. (2011) associaram a nutrição à atividade física e à autoestima e Silva et al. (2006) abordaram a nutrição como coadjuvante da intervenção, ao passo que o tema principal foi o controle de saúde dos indivíduos.

Os estudos realizados no território envolveram grupos que já existiam - três contaram com a participação de idosos em atividade de extensão universitária (ALENCAR; BARROS JÚNIOR; CARVALHO, 2008; CARVALHO; FONSECA; PEDROSA, 2004; CERVATO et al., 2005) e um com usuários de um centro poliesportivo (BUENO et al., 2011) - e grupos formados exclusivamente para a realização do estudo, com destaque para o desenvolvido por Santos et al. (2002) que, apesar de envolver usuários de UBS, teve como objetivo avaliar a intervenção na perspectiva territorial do serviço.

Ressaltam-se, nesse espaço, a diversidade da responsabilidade de execução da intervenção educativa e o envolvimento de estudantes estagiários e residente de nutrição. O papel da nutrição foi preponderante e exclusivo em dois estudos e, 
nos demais, foi coparticipante com atividade física em quatro intervenções, com outras demandas da saúde em três, com aspectos psicossociais em um e com aspectos sócio-políticos em um. Apenas um estudo, dentre os 28 selecionados, foi realizado em ambiente de trabalho. Baena et al. (2011) desenvolveram uma intervenção, mediada por nutricionista, com trabalhadores de plataforma marítima, seus familiares e a equipe da cozinha da plataforma. A atividade física teve papel coadjuvante na intervenção analisada.

Observa-se que apesar de a nutrição ter sido tema em todas as intervençôes, nem sempre o nutricionista era o executor da atividade educativa.

\section{Estruturação e base teórica}

A abordagem individual está presente em apenas seis estudos, sendo três relacionados ao tratamento de situações crônicas, como obesidade (CRUZ; MORIMOTO, 2004), síndrome metabólica (BUSNELLO et al., 2011) e hepatite C (LIMA et al., 2011) e outros três relacionados a intervenção com população obesa que praticava atividade física (MENDONÇA; LOPES, 2012), idosos que frequentavam curso de extensão universitária (CERVATO et al., 2005) e voluntários com alto risco metabólico (ALMEIDA-PITITTO et al., 2012).

Em contrapartida, as atividades envolvendo grupos se sobressaem. Dos 23 estudos encontrados com essa estruturação, quatro avaliaram treinamento (DAVANÇO; TADDEI; GAGLIANONE, 2004; GAGLIANONE et al., 2006; SANTOS et al., 2005, 2002); três referiam-se a atividades em ambulatório (ATTUX et al., 2011; FELIPPE et al., 2011; SILVA et al., 2006); cinco em escolas (BOOG, 2010; FERNANDES et al., 2009; FUJIMORI et al., 2008; GABRIEL; SANTOS; VASCONCELOS, 2008; VARGAS et al., 2011); seis trabalhavam com atividades abertas (ALENCAR; BARROS JÚNIOR; CARVALHO, 2008; BOEHS et al., 2005; BUENO et al., 2011; CARVALHO; FONSECA; PEDROSA, 2004; CERVATO et al., 2005; MENDONÇA; LOPES, 2012); quatro envolveram voluntários da comunidade (ALMEIDA-PITITTO et al., 2012; JAIME et al., 2007; LESSA; DEVINCENZI; SIGULEM, 2003; SANTOS et al., 2005) e um teve foco no local de trabalho (BAENA et al., 2011).

De modo geral, os objetivos das intervenções convergem para o enfrentamento da dupla carga de doença que caracteriza a situação alimentar e nutricional 
vigente. A prevenção e o tratamento de doenças crônicas e a desnutrição estão

relacionados aos objetivos das intervenções, bem como a promoção da saúde da criança, do adulto e do idoso.

As estratégias de intervenção encontradas foram descritas como consulta, visita domiciliar, palestra, encontro de grupo, curso e disciplina. Para as atividades educativas, foram utilizados os recursos: filmes, jogos, dinâmicas, brincadeiras, cartazes, fantoches, histórias, maquetes e degustação de alimentos. Segundo apresentado por alguns estudos, a duração média de cada intervenção foi de 15 minutos a duas horas, variando de um a 18 encontros que aconteceram, com mais frequência, mensalmente.

O referencial teórico mais comumente apontado pelos autores foi a abordagem social cognitiva e a dialógica de Paulo Freire. Entretanto, a maioria dos estudos não evidenciou o processo educativo, assim como não colocou em assunção um modelo teórico-educativo que sustentasse tais ações.

As intervençôes no ambiente escolar (tabela 2) abordaram aspectos cognitivos e atitudinais do comportamento alimentar, tendo por objetivo a mudança das práticas alimentares.

Tabela 2. Estruturação e base teórica de intervenções realizadas no ambiente escolar. São Paulo, 2013

\begin{tabular}{|c|c|c|c|c|}
\hline $1^{\circ}$ autor & Objetivo & Bases teóricas & Avaliação & Resultados \\
\hline Boog & $\begin{array}{l}\text { Incentivar o consumo } \\
\text { de alimentos regionais }\end{array}$ & $\begin{array}{c}\text { Conceitos da } \\
\text { promoção da } \\
\text { saúde, nutrição } \\
\text { comunitária e EAN } \\
\text { problematizadora }\end{array}$ & $\begin{array}{c}\text { Receptividade } \\
\text { do programa e } \\
\text { participação nas } \\
\text { atividades }\end{array}$ & $\begin{array}{l}\text { Impacto positivo } \\
\text { no consumo dos } \\
\text { alimentos }\end{array}$ \\
\hline Davanço & $\begin{array}{l}\text { Mudanças no } \\
\text { conhecimento, } \\
\text { atitudes e práticas }\end{array}$ & $\begin{array}{c}\text { Teoria Social } \\
\text { Cognitiva e Piaget }\end{array}$ & $\begin{array}{l}\text { Conhecimento, } \\
\text { atitudes e práticas } \\
\text { alimentares }\end{array}$ & $\begin{array}{c}\text { Melhora das variáveis } \\
\text { analisadas }\end{array}$ \\
\hline Fernandes & $\begin{array}{l}\text { Aumentar o } \\
\text { conhecimento }\end{array}$ & $\begin{array}{l}\text { Ferramenta do } \\
\text { guia da pirâmide } \\
\text { alimentar, com } \\
\text { processo educacional } \\
\text { interativo e dinâmico }\end{array}$ & $\begin{array}{c}\text { Consumo alimentar } \\
\text { e avaliação } \\
\text { antropométrica }\end{array}$ & $\begin{array}{c}\text { Melhora no consumo } \\
\text { alimentar }\end{array}$ \\
\hline
\end{tabular}

continua... 


\begin{tabular}{|c|c|c|c|c|}
\hline $1^{\circ}$ autor & Objetivo & Bases teóricas & Avaliação & Resultados \\
\hline Fujimori & $\begin{array}{l}\text { Avaliar a percepção } \\
\text { sobre o aleitamento } \\
\text { materno }\end{array}$ & $\begin{array}{c}\text { Crianças informadas } \\
\text { se tornam adultos } \\
\text { com maior capacidade } \\
\text { de escolha }\end{array}$ & $\begin{array}{l}\text { Conhecimento sobre } \\
\text { amamentação }\end{array}$ & $\begin{array}{c}\text { Melhora das variáveis } \\
\text { analisadas }\end{array}$ \\
\hline Gabriel & $\begin{array}{c}\text { Mudanças de práticas } \\
\text { alimentares }\end{array}$ & $\begin{array}{l}\text { Nível cognitivo } \\
\text { esperado do público- } \\
\text { alvo e assessoria } \\
\text { de nutricionista e } \\
\text { pedagogo }\end{array}$ & $\begin{array}{c}\text { Práticas alimentares } \\
\text { e avaliação } \\
\text { antropométrica }\end{array}$ & $\begin{array}{c}\text { Melhora nas práticas } \\
\text { alimentares }\end{array}$ \\
\hline Gaglianone & $\begin{array}{c}\text { Mudança de práticas } \\
\text { dos alunos e aumentar } \\
\text { conhecimento/atitude } \\
\text { dos professores }\end{array}$ & $\begin{array}{c}\text { Teoria Social } \\
\text { Cognitiva e Piaget }\end{array}$ & $\begin{array}{c}\text { Práticas alimentares } \\
\text { dos alunos e } \\
\text { conhecimento/atitude } \\
\text { dos professores }\end{array}$ & $\begin{array}{c}\text { Melhora das variáveis } \\
\text { analisadas }\end{array}$ \\
\hline Vargas & $\begin{array}{c}\text { Mudanças nas } \\
\text { práticas alimentares }\end{array}$ & $\begin{array}{c}\text { Identificação prévia } \\
\text { de temáticas de } \\
\text { interesse }\end{array}$ & Consumo alimentar & $\begin{array}{c}\text { Melhora nas práticas } \\
\text { alimentares }\end{array}$ \\
\hline
\end{tabular}

Fonte: elaboração própria.

Dos sete estudos desenvolvidos em ambiente escolar, destacam-se dois (DAVANÇO; TADDEI; GAGLIANONE, 2004; GAGLIANONE et al., 2006) que escolheram a Teoria Social Cognitiva e a Teoria de Piaget para a intervenção realizada, apresentando-as de forma clara.

Nos estudos realizados nos serviços de saúde (tabela 3), verificou-se que os objetivos das intervençôes estavam fundamentados na necessidade de aumentar a adesão ao tratamento proposto. Porém, por mais que essa ideia remetesse à análise de aspectos cognitivos e comportamentais, foram os dados antropométricos e bioquímicos que receberam maior ênfase como ferramentas de avaliação. De qualquer forma, observou-se que apenas dois estudos (ARAUJO; FIGUEIREDO; D’AVILA, 2010; FAZIO et al., 2011) não atingiram seus objetivos, ao passo que não foram encontradas diferenças após intervenção. 
São Paulo, 2013

\begin{tabular}{|c|c|c|c|c|}
\hline $1^{\circ}$ autor & Objetivo & Bases teóricas & Avaliação & Resultados \\
\hline Adde & $\begin{array}{c}\text { Melhorar o estado } \\
\text { nutricional }\end{array}$ & $\begin{array}{c}\text { Necessidade de } \\
\text { verificar se a } \\
\text { intervenção produz } \\
\text { resultado }\end{array}$ & $\begin{array}{c}\text { Avaliação } \\
\text { antropométrica e } \\
\text { dados clínicos }\end{array}$ & $\begin{array}{c}\text { Melhora do estado } \\
\text { nutricional }\end{array}$ \\
\hline Araujo & $\begin{array}{c}\text { Aumentar } \\
\text { conhecimento } \\
\text { e melhorar o } \\
\text { comportamento } \\
\text { alimentar }\end{array}$ & $\begin{array}{c}\text { Ensino-aprendizagem } \\
\text { sobre temáticas } \\
\text { específicas }\end{array}$ & $\begin{array}{c}\text { Conhecimento e } \\
\text { dados bioquímicos }\end{array}$ & Não houve diferença \\
\hline Attux & $\begin{array}{c}\text { Melhorar a saúde } \\
\text { mental e a adequação } \\
\text { do peso }\end{array}$ & $\begin{array}{l}\text { Programa de } \\
\text { Esquizofrenia }\end{array}$ & $\begin{array}{c}\text { Avaliação } \\
\text { antropométrica }\end{array}$ & $\begin{array}{l}\text { Pequena diferença } \\
\text { antropométrica }\end{array}$ \\
\hline Busnello & $\begin{array}{c}\text { Avaliar adesão } \\
\text { a tratamento } \\
\text { dietoterápico e } \\
\text { impacto na melhora } \\
\text { clínica }\end{array}$ & $\begin{array}{l}\text { Manual de Orientação } \\
\text { Nutricional para } \\
\text { Pacientes com } \\
\text { Síndrome Metabólica }\end{array}$ & $\begin{array}{c}\text { Avaliação } \\
\text { antropométrica, } \\
\text { consumo alimentar, } \\
\text { dados bioquímicos e } \\
\text { motivação }\end{array}$ & $\begin{array}{l}\text { Pacientes mais } \\
\text { motivados } \\
\text { responderam melhor } \\
\text { ao tratamento }\end{array}$ \\
\hline Cruz & $\begin{array}{l}\text { Avaliar protocolo de } \\
\text { atendimento }\end{array}$ & $\begin{array}{l}\text { Guia alimentar da } \\
\text { pirâmide americana }\end{array}$ & $\begin{array}{c}\text { Alimentação e queixas } \\
\text { dos pacientes }\end{array}$ & $\begin{array}{l}\text { Protocolo permitiu } \\
\text { a adesão pela baixa } \\
\text { incidência de } \\
\text { complicaçóes }\end{array}$ \\
\hline Fazio & $\begin{array}{l}\text { Conhecer o consumo } \\
\text { dietético e ganho de } \\
\text { peso }\end{array}$ & $\begin{array}{l}\text { Hábito alimentar } \\
\text { saudável parte } \\
\text { de um consumo } \\
\text { adequado em macro e } \\
\text { micronutrientes }\end{array}$ & $\begin{array}{c}\text { Registro de } \\
\text { prontuários, } \\
\text { consumo alimentar e } \\
\text { antropométrico }\end{array}$ & Não houve diferença \\
\hline Felippe & $\begin{array}{l}\text { Avaliar mudança de } \\
\text { hábitos alimentares }\end{array}$ & Sem especificação & $\begin{array}{c}\text { Avaliação } \\
\text { antropométrica e } \\
\text { consumo alimentar }\end{array}$ & $\begin{array}{l}\text { Não houve diferença } \\
\text { no peso, mas melhora } \\
\text { no consumo alimentar }\end{array}$ \\
\hline Lima & $\begin{array}{c}\text { Compreender os } \\
\text { significados atribuídos } \\
\text { à doença e ao } \\
\text { tratamento }\end{array}$ & $\begin{array}{l}\text { Necessidade de ouvir } \\
\text { e compreender a } \\
\text { realidade do paciente }\end{array}$ & $\begin{array}{c}\text { Dimensão qualitativa } \\
\text { da doença e do } \\
\text { tratamento }\end{array}$ & $\begin{array}{l}\text { Processo dialógico } \\
\text { produz mudanças na } \\
\text { vida do paciente }\end{array}$ \\
\hline Silva & $\begin{array}{c}\text { Melhorar os índices } \\
\text { de pressão arterial e } \\
\text { glicemia }\end{array}$ & $\begin{array}{l}\text { Estratégias de } \\
\text { ações educativas e } \\
\text { terapêuticas }\end{array}$ & $\begin{array}{l}\text { Adesão e pressão } \\
\text { arterial e glicemia }\end{array}$ & $\begin{array}{c}\text { Melhora das variáveis } \\
\text { analisadas }\end{array}$ \\
\hline
\end{tabular}

Fonte: elaboração própria. 
Os estudos realizados no território (tabela 4) referiram-se a um conjunto diversificado de intervenções que explicitaram os princípios teóricos do conteúdo abordado. Por outro lado, incluíram avaliações de comentários, depoimentos e vivências, visando identificar aspectos relacionados a determinado comportamento.

Tabela 4. Estruturação e base teórica de intervenções realizadas no território. São Paulo, 2013

\begin{tabular}{|c|c|c|c|c|}
\hline $1^{\circ}$ autor & Objetivo & Bases teóricas & Avaliação & Resultados \\
\hline Alencar & $\begin{array}{l}\text { Refletir sobre as } \\
\text { vivências de sala } \\
\text { de aula }\end{array}$ & $\begin{array}{l}\text { Educação crítica } \\
\text { de Paulo Freire }\end{array}$ & $\begin{array}{c}\text { Observação } \\
\text { participante e } \\
\text { entrevista }\end{array}$ & $\begin{array}{c}\text { Discussão das } \\
\text { dimensões } \\
\text { sócio-políticas da } \\
\text { alimentação }\end{array}$ \\
\hline $\begin{array}{l}\text { Almeida- } \\
\text { Pititto }\end{array}$ & $\begin{array}{c}\text { Mudanças } \\
\text { comportamentais }\end{array}$ & $\begin{array}{c}\text { Recomendaçóes da } \\
\text { OMS }\end{array}$ & $\begin{array}{c}\text { Índices de } \\
\text { pressão arterial } \\
\text { e parâmetros } \\
\text { metabólicos }\end{array}$ & $\begin{array}{c}\text { Melhora do perfil } \\
\text { cardiovascular }\end{array}$ \\
\hline Boehs & $\begin{array}{l}\text { Apoiar a família } \\
\text { cuidadora de } \\
\text { crianças com } \\
\text { desnutriçãa }\end{array}$ & $\begin{array}{c}\text { O brincar para } \\
\text { fortalecer os laços }\end{array}$ & $\begin{array}{l}\text { Comentários e } \\
\text { depoimentos }\end{array}$ & $\begin{array}{l}\text { Aprendizado } \\
\text { mútuo e a } \\
\text { valorização da } \\
\text { família }\end{array}$ \\
\hline Bueno & $\begin{array}{l}\text { Avaliar a adesão } \\
\text { e evolução } \\
\text { antropométrica }\end{array}$ & $\begin{array}{l}\text { EAN que articula } \\
\text { atitudes e práticas } \\
\text { alimentares }\end{array}$ & $\begin{array}{c}\text { Adesão e avaliação } \\
\text { antropométrica }\end{array}$ & $\begin{array}{l}\text { Baixa adesão, } \\
\text { redução de peso } \\
\text { corporal }\end{array}$ \\
\hline Carvalho & $\begin{array}{c}\text { Aumentar } \\
\text { conhecimento, } \\
\text { atenção e cuidado }\end{array}$ & $\begin{array}{c}\text { Educação } \\
\text { pela prática, } \\
\text { modificação de } \\
\text { comportamento } \\
\text { pela intervenção } \\
\text { ativa }\end{array}$ & $\begin{array}{l}\text { Conhecimento } \\
\text { e consumo } \\
\text { alimentar }\end{array}$ & $\begin{array}{c}\text { Melhora } \\
\text { das variáveis } \\
\text { analisadas }\end{array}$ \\
\hline Cervato & $\begin{array}{l}\text { Mudanças } \\
\text { no consumo } \\
\text { alimentar }\end{array}$ & $\begin{array}{l}\text { Conscientização } \\
\text { de hábito } \\
\text { alimentar, } \\
\text { mobilizando- } \\
\text { os para o } \\
\text { autocuidado }\end{array}$ & $\begin{array}{l}\text { Conhecimento } \\
\text { e consumo } \\
\text { alimentar }\end{array}$ & $\begin{array}{c}\text { Melhora } \\
\text { das variáveis } \\
\text { analisadas }\end{array}$ \\
\hline
\end{tabular}

continua... 


\begin{tabular}{|c|c|c|c|c|}
\hline $1^{\circ}$ autor & Objetivo & Bases teóricas & Avaliação & Resultados \\
\hline Jaime & $\begin{array}{c}\text { Incentivar o } \\
\text { consumo de frutas } \\
\text { e hortaliças }\end{array}$ & $\begin{array}{l}\text { Diagnóstico } \\
\text { motivacional e } \\
\text { informacional }\end{array}$ & $\begin{array}{l}\text { Aquisição de } \\
\text { alimentos }\end{array}$ & $\begin{array}{c}\text { Aumento do } \\
\text { consumo de frutas } \\
\text { e hortaliças }\end{array}$ \\
\hline Lessa & $\begin{array}{c}\text { Desenvolver } \\
\text { modelo de atenção } \\
\text { na prevenção } \\
\text { de carências } \\
\text { nutricionais }\end{array}$ & $\begin{array}{c}\text { Programa com } \\
\text { ações de educação } \\
\text { e promoção em } \\
\text { saúde }\end{array}$ & $\begin{array}{c}\text { Avaliação } \\
\text { antropométrica, } \\
\text { dados } \\
\text { bioquímicos, } \\
\text { tempo de } \\
\text { aleitamento } \\
\text { materno }\end{array}$ & $\begin{array}{c}\text { Redução da } \\
\text { prevalência de } \\
\text { anemia e aumento } \\
\text { do tempo de } \\
\text { aleitamento }\end{array}$ \\
\hline Mendonça & $\begin{array}{c}\text { Promover a } \\
\text { qualidade de vida }\end{array}$ & $\begin{array}{l}\text { Materiais do } \\
\text { Ministério da } \\
\text { Saúde }\end{array}$ & $\begin{array}{c}\text { Avaliação } \\
\text { antropométrica, } \\
\text { pressão arterial } \\
\text { e consumo } \\
\text { alimentar }\end{array}$ & $\begin{array}{c}\text { Melhora } \\
\text { das variáveis } \\
\text { analisadas }\end{array}$ \\
\hline Santos & $\begin{array}{l}\text { Mudanças de } \\
\text { conhecimentos e } \\
\text { prática profissional }\end{array}$ & $\begin{array}{c}\text { Atenção Integral } \\
\text { às doenças } \\
\text { prevalentes na } \\
\text { infância, da OMS } \\
\text { e UNICEF }\end{array}$ & $\begin{array}{l}\text { Conhecimento/ } \\
\text { prática profissional } \\
\text { e materna e } \\
\text { comportamento } \\
\text { alimentar infantil }\end{array}$ & $\begin{array}{c}\text { Melhora } \\
\text { das variáveis } \\
\text { analisadas }\end{array}$ \\
\hline Santos & $\begin{array}{l}\text { Efetividade de } \\
\text { treinamento de } \\
\text { líderes }\end{array}$ & $\begin{array}{l}\text { Treinamento da } \\
\text { OMS e UNICEF, } \\
\text { adaptado }\end{array}$ & $\begin{array}{c}\text { Práticas } \\
\text { alimentares e } \\
\text { dados bioquímicos }\end{array}$ & $\begin{array}{l}\text { Não houve } \\
\text { diferença }\end{array}$ \\
\hline
\end{tabular}

Fontes: OMS e UNICEF.

De modo geral, independentemente do cenário da pesquisa, foi possível verificar uma descrição detalhada do instrumento de coleta de dados e dos procedimentos de análise. Entretanto, a intervenção foi discutida de maneira sucinta e, geralmente, incompleta.

\section{Discussão}

A seleção de artigos publicados no período de 2000 a 2012 partiu da necessidade de identificar a configuração das intervenções realizadas antes da criação 
do Marco de Referência de Educação Alimentar e Nutricional para as Políticas Públicas, tido como um documento que orienta a realização de ações educativas transformadoras (BRASIL, 2012). Diante disso, compreende-se que estudos publicados no período pós-marco devam ser avaliados em outra perspectiva, ou seja, de uma intervenção mais inclusiva e problematizadora.

Por mais que tenha se averiguado que a produção do conhecimento de educação nutricional está concentrada nas universidades públicas do estado de São Paulo, são as instituições de outros estados as responsáveis por conduzirem suas intervenções ao encontro dos ideais do marco de referência, mesmo antes de sua criação. Ao se apropriarem da abordagem qualitativa, os estudos realizados na Bahia (LIMA et al., 2011), no Rio Grande do Sul (SANTOS et al., 2002) e no Piauí (ALENCAR; BARROS JÚNIOR; CARVALHO, 2008) propõem que a prática alimentar seja analisada pela "perspectiva da promoção da saúde, corresponsabilização, autonomia, autocuidado e reconhecimento da alimentação como direito social e exercício da cidadania" (BRASIL, 2012, p. 3).

Espera-se, pois, que, com o uso do marco como referência teórica, essas instituições que vêm trabalhando com pesquisa qualitativa possam fortalecer seus estudos, sobretudo os de intervenção, bem como ampliarem o escopo teórico da educação nutricional, favorecendo a troca de conhecimento e experiências entre instituições e regiōes do país.

Quanto aos contextos de intervenção e atores envolvidos, verifica-se que o serviço de saúde foi o espaço em que o nutricionista mais apareceu como o único responsável pela intervenção. Em contrapartida, aqueles desenvolvidos no ambiente escolar e no território não especificaram a responsabilidade ou tiveram o nutricionista atuando de maneira compartilhada com outros profissionais; ou ainda, envolveram atores em formação, como estudante e residente de Nutrição.

A expectativa era encontrar um número maior de artigos que envolvesse o nutricionista na intervenção, em função da importância que a educação nutricional tem como atribuição desse profissional (CFN, 2005). Diante dos resultados, levanta-se a suposição de que poucos nutricionistas estejam atuando nessas áreas ou as atividades de educação nutricional desenvolvidas por eles não têm acontecido de forma sistematizada e os conhecimentos não têm sido divulgados. Compreende-se que isso seja uma consequência histórica da falta de uma definição clara e objetiva da EAN e da insuficiência de respaldo teórico 
para o embasamento de suas práticas, tendo, pois, constituído inquietações que regeram a elaboração do marco (BRASIL, 2012).

Também se baseando na constatação de não correspondência à expectativa fato que compromete a ideia de reforçar a importância da EAN no contexto da Promoção da Saúde - o marco amplia a responsabilidade das ações educativas sobre alimentação e nutrição para outros profissionais da saúde, buscando aumentar a ocorrência e a visibilidade das experiências em diferentes campos de atuação (BRASIL, 2012). A partir disso, infere-se que a ampliação para a atuação, advinda do marco, deve-se ao fato de que nutricionistas não vinham desenvolvendo as práticas de forma suficientemente acessíveis às necessidades e demandas do campo.

Contudo, mesmo frente a esses apontamentos, ainda há de se esperar que os profissionais não nutricionistas planejem e executem suas ações de EAN articulando seus conhecimentos ao suporte técnico-pedagógico oferecido por aqueles que, com ou sem o marco, continuarão sendo os atores com formação acadêmica específica à compreensão da ciência da Nutrição, sobretudo ao entendimento das dimensões sócio-psico-culturais emergentes da relação entre o homem e o alimento, no contexto da realização do Direito Humano à Alimentação Adequada e da garantia da Segurança Alimentar e Nutricional: os nutricionistas.

$\mathrm{O}$ achado referente ao ambiente escolar assemelha-se à revisão sistemática realizada por Pereira, Scagliusi e Batista (2011), que incluiu apenas dez estudos sobre tendências metodológicas no tocante às pesquisas de educação nutricional. Segundo os autores, o pequeno número de trabalhos deve-se à prática ainda incomum de publicar intervenções. Acrescentam também que, apesar da incipiência de publicação, são desenvolvidas atividades sobre o tema nos âmbitos nacional e internacional.

Souza et al. (2011) reforçam a importância da sistematização e da publicação de informações de estudos de intervenção, como a estratégia e os materiais de apoio didático, visto que essas servem como referência à prática profissional quando seus resultados são positivos.

Ao observar os conteúdos referidos nas intervenções, constata-se que a abordagem nutricional é predominante justamente nos serviços de saúde, onde o nutricionista mais tem atuado como responsável pela condução de ações educativas. Os estudos desenvolvidos no ambiente escolar e de trabalho 
também abordaram nutrição, porém, associando-a, na maioria das vezes, ao tema atividade física. Já os realizados no território destacaram-se por envolverem junto à nutrição, além da atividade física, temas mais amplos como aspectos sociopolíticos e psicossociais. Aponta-se que, talvez, essa abordagem ampliada possa estar sustentada no fato de os profissionais estarem no contexto de vida dos indivíduos e das comunidades, o que gera a necessidade de envolver outros aspectos além da nutrição.

De modo geral, as intervenções desenvolvidas nos serviços de saúde tiveram como pretensão o tratamento de doenças, diferentemente do que se verificou nos territórios, nas escolas e no trabalho, cujo intuito era promover saúde e alimentação saudável. Nesse sentido, o fato de se ter encontrado mais nutricionistas participando de intervençôes nos serviços de saúde é reforçado pela ideia estabelecida no marco ao referir que, em um contexto em que a EAN é compreendida como recurso terapêutico de cuidado e cura de agravo, cabe ao nutricionista a responsabilidade pela intervenção. Vale ressaltar também que a perspectiva da alimentação saudável, presente nos demais cenários, é entendida, no marco, como uma estratégia de enfrentamento dos novos desafios no campo da saúde, prevista a todos os profissionais da área, inclusive aos nutricionistas da saúde, à medida que a prática de hábitos alimentares saudáveis é inerente a qualquer contexto que vise promover saúde (BRASIL, 2012).

Boog (2013) refere que as açôes educativas de alimentação e nutrição devem ser procedidas de um diagnóstico educativo, diferente do diagnóstico de saúde como os apresentados na maioria dos artigos estudados. Cervato-Mancuso (2011) também sustenta essa ideia, destacando a importância da estruturação de um planejamento com objetos, recursos, atividades, efeitos e contexto da intervenção, com clareza do grupo populacional para o qual a ação será dirigida. Nesse sentido, dentre os estudos apresentados nesta revisão, aqueles referentes às intervenções em escolas apresentaram maior detalhamento das açôes, com destaque para os que explicitaram o referencial teórico utilizado, fornecendo, assim, mais elementos para a compreensão do processo educativo testado na pesquisa.

Apesar de a maioria dos estudos ter descrito de maneira clara e objetiva a metodologia de análise, a explicação insuficiente da intervenção não permitiu o detalhamento do método educativo investigado. Tal constatação converge com os resultados encontrados por Leão et al. (2011) em revisão sistemática sobre 
intervenções nutricionais na remissão do diagnóstico de síndrome metabólica, que evidenciou a falta de informações dos desenhos aplicados nas intervenções. Além disso, os autores verificaram uma limitação quantitativa e uma heterogeneidade desses estudos em relação ao tema. Outra revisão sistemática, realizada por Sartorelli, Franco e Cardoso (2006), encontrou apenas nove publicações referentes a prevenção primária de diabetes mellitus tipo 2 a partir da orientação nutricional, havendo prevalência de intervenções direcionadas à alimentação saudável isolada ou associada à prática de atividades físicas, assim como constatado na presente revisão.

De maneira geral, as duas revisōes sistemáticas supracitadas se assemelham a esta revisão em relação à descrição dos programas de intervenção, pautadas em informações sobre características dietéticas, relacionadas a energia, nutrientes e consumo de grupos alimentares. As tendências metodológicas que nortearam as práticas dos estudos analisados coadunam com os achados de Santos (2012), que situa tais ações em modelos tradicionais, baseados na transmissão de informação, apesar de várias referências a Paulo Freire e à educação popular em saúde. Segundo o autor, é necessário fortalecer o processo de formação profissional e o investimento na produção de conhecimento do campo de alimentação e nutrição.

Dentre as características descritas pelos estudos, está a definição da população estudada. Algumas experiências educativas abrangeram diferentes grupos, incluindo grupos secundários, representados por pessoas que recebiam orientações direcionadas à mudança de condutas de outros indivíduos, a exemplo do que aconteceu na atuação de professores e líderes comunitários. Os professores têm sido objeto de vários estudos, em função da importância de seu papel de educadores nas escolas que, como tal, promove troca de informações que pode vir a incluir o tema da alimentação (ASSAO; CERVATO-MANCUSO, 2008; IULIANO; CERVATO-MANCUSO; GAMBARDELLA, 2009).

Segundo Cervato-Mancuso (2011), as intervenções de educação em nutrição possuem uma estrutura teórico-metodológica de planejamento constituída pelas fases de concepção, formulação, implementação e avaliação, sendo que esta última deve ser realizada desde a concepção até a finalização da intervenção. Assim, avaliar significa fazer uma análise crítica, objetiva e sistemática das realizações e dos resultados fundamentais, para evidenciar as experiências positivas que podem ser replicadas em outro espaço e tempo. 
As avaliações das intervenções dos estudos analisados seguiram a perspectiva de seus objetivos; assim, a maioria remete aos indicadores quantitativos, sobretudo para análise de consumo alimentar e verificação antropométrica. Novamente, o cenário do território diferenciou-se dos outros, pois realizaram avaliaçôes por meio de comentários, depoimentos, entrevista, observação e outros, ampliando a visão para além dos valores numéricos.

Essa constatação assemelha-se aos achados de Boog (2013), que refere que grande parte dos estudos no campo restringe seus resultados à mensuração de conhecimentos sobre nutrição ou à mudança de práticas alimentares. A autora aponta a necessidade de estudos que ampliem a perspectiva de avaliação para dados não mensuráveis, uma vez que o fenômeno da alimentação é complexo e envolve sentimentos, valores e representações sobre os alimentos.

Por fim, entendendo que o aumento das ações educativas em grupo é uma tendência de atenção em saúde, fazem-se necessários estudos com esse enfoque. Nesse sentido, pensar em intervenção grupal é pensar em prática social, em participação coletiva e em construção compartilhada que exigem do profissional preparo e habilidade para o planejamento, assim como o desenvolvimento e a avaliação da intervenção educativa em nutrição, que podem ser respaldadas pelo uso do marco de referência.

\section{Considerações finais}

O intuito das intervençôes tem mudado ao longo das últimas décadas. Se antes se centrava na distribuição de alimentos e no aumento do conhecimento, hoje foca na mudança do comportamento individual, porém ainda associando-o ao saber científico. Compreendendo o comportamento alimentar como resultado de relaçôes sociais e históricas, as intervençôes educativas precisam superar suas raízes biomédicas, partindo de uma responsabilidade individual para a responsabilidade coletiva, e de um conhecimento científico para a construção de um novo conhecimento em que todos participem.

As intervenções que buscam alternativas para o quadro epidemiológico atual representam uma oportunidade para a atuação do nutricionista. Entretanto, essas intervenções vêm sendo realizadas sem a presença do profissional ou muitas delas não têm sido devidamente documentadas e avaliadas. 
Considerando que a EAN propõe um compartilhamento de responsabilidade, destaca-se a necessidade não só de descrever a equipe multiprofissional responsável pela ação e/ou programa, mas, também, de evidenciar o papel de cada elemento da equipe durante o processo educativo.

Diante do exposto, é possível evidenciar que as intervenções nutricionais, mesmo aquelas com componente educativo, apresentam descrição metodológica da educação superficial, que não fornece elementos suficientes para a reprodução do estudo ou da sua implementação em outros cenários de prática.

As bases teóricas que regem tais ações, apesar de serem reportadas por diferentes autores, não foram incorporadas nos procedimentos metodológicos dos artigos analisados. Contudo, espera-se que intervenções futuras fortaleçam suas bases, dado a disponibilidade do Marco de Referência de Educação Alimentar e Nutricional para as Políticas Públicas. Por se tratar de um documento de reflexão e orientação da prática, sua utilização pode se dar em diversos contextos, inclusive nos verificados neste estudo - escola, serviços de saúde, território e ambiente de trabalho -, na expectativa de que sejam produzidos outros indicadores de avaliação, como o grau de vínculo e de autonomia em saúde, que englobem todas as dimensões que constituem a relação entre o alimento e o homem e deste com o profissional-educador no bojo da promoção da saúde. ${ }^{1}$

\section{Referências}

ADDE, F. V.; RODRIGUES, J. C.; CARDOSO, A. L. Seguimento nutricional de pacientes com fibrose cística: papel do aconselhamento nutricional. Jornal de Pediatria, v. 80, n. 6, p. 475-482, dez. 2004.

ALENCAR, M. S. S.; BARROS JÚNIOR, F. O.; CARVALHO, C. M. R. G. Os aportes sócio-políticos da educação nutricional na perspectiva de um envelhecimento saudável. Revista de Nutrição, v. 21, n. 4, p. 369-381, ago. 2008.

ALMEIDA-PITITTO, B. et al. A behavioral intervention in a cohort of Japanese-Brazilians at high cardiometabolic risk. Revista de Saúde Pública, v. 46, n. 4, p. 602-609, ago. 2012. ARAujO, L. P. R.; Figueiredo, A. E. P. L.; D’AVILA, D. O. L. Avaliação de programa de ensino-aprendizagem sobre metabolismo de cálcio e fósforo para pacientes em hemodiálise. Revista da Escola de Enfermagem da USP, v. 44, n. 4, p. 928-932, dez. 2010.

ASSAO, T. Y.; CERVATO-MANCUSO, A. M. Alimentação saudável: percepções dos educadores de instituiçóes infantis. Revista brasileira de crescimento e desenvolvimento bumano, v. 18, n. 2, p. 126-134, 2008. 
ATTUX, C. et al. The effectiveness of a non-pharmacological intervention for weight gain management in severe mental disorders : results from a national multicentric study. Revista Brasileira de Psiquiatria, v. 33, n. 2, p. 117-121, 2011.

BAENA, C. P. et al. Impacto de um programa piloto de promoção da saúde para trabalhadores marítimos de rebocadores. Revista Brasileira de Saúde Ocupacional, v. 36, n. 124, p. 288-296, dez. 2011.

BOEHS, A. E. et al. Famílias com crianças desnutridas: os desafios para trabalhar em grupos. Texto \& Contexto - Enfermagem, v. 14, n. 2, p. 287-292, jun. 2005.

BOOG, M. C. F. Educação nutricional: passado, presente e futuro. Revista de Nutrição, v. 10, n. 1, p. 5-19, 1997.

- Atuação do nutricionista em saúde pública na promoção da alimentação saudável. Revista Ciência \& Saúde, v. 1, n. 1, p. 33-42, 2008.

. Programa de educação nutricional em escola de ensino fundamental de zona rural. Revista de Nutrição, v. 23, n. 6, p. 1005-1017, dez. 2010.

. Histórico da Educação Alimentar e Nutricional no Brasil. In: DIEZ-GARCIA; CERVATO-MANCUSO (Orgs.). Mudanças alimentares e educação nutricional. 1. ed. Rio de Janeiro: Guanabara Koogan, 2011. p. 66-73.

Educação em Nutrição: integrando experiências. 1. ed. Campinas-SP: Komedi, 2013. p. 286.

BRASIL. Ministério do Desenvolvimento Social e Combate à Fome. Secretaria Nacional de Segurança Alimentar e Nutricional. Marco de Referência de Educação Alimentar e Nutricional para as Políticas Públicas. Brasília-DF: Ministério da Saúde, 2012.

BUENO, J. M. et al. Educação alimentar na obesidade: adesão e resultados antropométricos. Revista de Nutrição, v. 24, n. 4, p. 575-584, ago. 2011.

BUSNELLO, F. M. et al. Intervenção nutricional e o impacto na adesão ao tratamento em pacientes com síndrome Metabólica. Arquivos Brasileiros de Cardiologia, v. 97, n. 3, p. 217224, set. 2011.

CARVAlHO, C. M. R. G.; FONSECA, C. C. C.; PEDROSA, J. I. Educação para a saúde em osteoporose com idosos de um programa universitário: repercussões. Cadernos de Saúde Pública, v. 20, n. 3, p. 719-726, jun. 2004.

CASTRO, C.; PELIANO, A. M. Novos alimentos, velhos hábitos e o espaço para ações educativas. In: CASTRO; COIMBRA (Orgs.). O problema alimentar no Brasil. 1. ed. São Paulo: Unicamp, 1985. p. 195-213.

CASTrO, C. M.; COIMBRA, M. O problema alimentar no Brasil. 1. ed. São Paulo: Unicamp, 1985. p. 213. 
CERVATO, A. M. et al. Educação nutricional para adultos e idosos: uma experiência positiva em Universidade Aberta para a Terceira Idade. Revista de Nutrição, v. 18, n. 1, p. 41-52, fev. 2005.

CERVATO-MANCUSO, A. M. Elaboração de um programa de educação nutricional. In: DIEZ-GARCIA; CERVATO-MANCUSO (Orgs.). Mudanças alimentares e educação nutricional. Rio de Janeiro: Guanabara Koogan, 2011. p. 187-197.

CONSELHO FEDERAL DE NUTRICIONISTAS. Resolução CFN nº 380/2005. Dispõe sobre a definição das áreas de atuação do nutricionista e suas atribuições, estabelece parâmetros numéricos de referência por área de atuação e dá outras providencias. Brasília: CFN, 2005.

CRUZ, M. R. R.; MORIMOTO, I. M. I. Intervenção nutricional no tratamento cirúrgico da obesidade mórbida: resultados de um protocolo diferenciado. Revista de Nutrição, v. 17, n. 2, p. 263-272, jun. 2004.

DAVANÇO, G. M.; TADDEI, J. A.; GAGLIANONE, C. P. Knowledge, attitudes and practices of teachers of basic cycle, exposed and non exposed to a Nutrition Education Course. Revista de Nutrição, v. 17, n. 2, p. 177-184, jun. 2004.

FAZIO, E. DE S. et al. Consumo dietético de gestantes e ganho ponderal materno após aconselhamento nutricional. Revista Brasileira de Ginecologia e Obstetrícia, v. 33, n. 2, p. 87-92, fev. 2011.

FELIPPE, F. et al. Qualidade da dieta de indivíduos expostos e não expostos a um programa de reeducação alimentar. Revista de Nutrição, v. 24, n. 6, p. 833-844, 2011.

FERNANDES, P. S. et al. Evaluating the effect of nutritional education on the prevalence of overweight/obesity and on foods eaten at primary schools. Jornal de Pediatria, p. 1-10, 4 ago. 2009.

FUJIMORI, M. et al. Percepção de estudantes do ensino fundamental quanto ao aleitamento materno e a influência da realização de palestras de educação em saúde. Jornal de Pediatria, v. 84, n. 3, p. 224-231, 30 jun. 2008.

GABRIEL, C. G.; SANTOS, M. V.; VASCONCELOS, F. A. G. DE. Avaliação de um programa para promoção de hábitos alimentares saudáveis em escolares de Florianópolis, Santa Catarina, Brasil. Revista Brasileira de Saúde Materno Infantil, v. 8, n. 3, p. 299-308, set. 2008.

GAGLIANONE, C. P. et al. Nutrition education in public elementary schools of São Paulo, Brazil: the Reducing Risks of Illness and Death in Adulthood project. Revista de Nutrição, v. 19, n. 3, p. 309-320, jun. 2006.

IUlianO, B. A.; CERVATO-MANCUSO, A. M.; GAMBARDElla, A. M. D. Educação nutricional em escolas de ensino fundamental do município de Guarulhos-SP. $O$ mundo da saúde, São Paulo, v. 33, n. 3, p. 264-272, 2009. 
JAIME, P. C. et al. Educação nutricional e consumo de frutas e hortaliças: ensaio comunitário controlado. Revista de Saúde Pública, v. 41, n. 1, p. 154-157, fev. 2007.

LEÃO, L. S. C. S. et al. Intervençōes nutricionais em Síndrome Metabólica: uma revisão sistemática. Arquivos Brasileiros de Cardiologia, v. 97, n. 3, p. 260-265, set. 2011.

LESSA, A. C.; DEVINCENZI, M. U.; SIGULEM, D. M. Comparação da situação nutricional de crianças de baixa renda no segundo ano de vida, antes e após a implantação de programa de atenção primária à saúde. Cadernos de Saúde Pública, v. 19, n. 2, p. 505-514, abr. 2003.

LIMA, C. M. C. et al. Reflexôes sobre o adoecer e mudanças dietéticas durante a assistência nutricional em pacientes com hepatite C. Physis: Revista de Saúde Coletiva, v. 21, n. 2, p. 647-662, 2011.

MENDONÇA, R. D.; LOPES, A. C. S. The effects of health interventions on dietary habits and physical measurements. Revista da Escola de Enfermagem da USP, v. 46, n. 3, p. 573-579, jun. 2012.

PEREIRA, P. R.; SCAGLIUSI, F. B.; BATISTA, S. H. S. S. Educação nutricional nas escolas: um estudo de revisão sistemática. Nutrire, v. 36, n. 3, p. 109-129, 2011.

SANTOS, I. S. et al. Avaliação da eficácia do aconselhamento nutricional dentro da estratégia do AIDPI (OMS/UNICEF). Revista Brasileira de Epidemiologia, v. 5, n. 1, p. 15-29, abr. 2002.

. Efetividade do aconselhamento nutricional da Pastoral da Criança sobre a variação de hemoglobina entre menores de seis anos de idade. Cadernos de Saúde Pública, v. 21, n. 1, p. $130-140$, fev. 2005.

SANTOS, L. A. S. O fazer educação alimentar e nutricional : algumas contribuições para reflexão. Ciência \& Saúde Coletiva, v. 17, n. 2, p. 453-462, 2012.

SARTORELLI, D. S.; FRANCO, L. J.; CARDOSO, M. A. Intervenção nutricional e prevenção primária do diabetes mellitus tipo 2: uma revisão sistemática. Cadernos de Saúde Pública, v. 22, n. 1, p. 7-18, jan. 2006.

SILVA, T. R. et al. Controle de diabetes Mellitus e hipertensão arterial com grupos de intervenção educacional e terapêutica em seguimento ambulatorial de uma Unidade Básica de Saúde. Saúde e Sociedade, v. 15, n. 3, p. 180-189, dez. 2006.

SOUZA, E. A. et al. Atividade física e alimentação saudável em escolares brasileiros: revisão de programas de intervenção. Cadernos de Saúde Pública, v. 27, n. 8, p. 1459-1471, ago. 2011. VARGAS, I. C. DA S. et al. Avaliação de programa de prevenção de obesidade em adolescentes de escolas públicas. Revista de Saúde Pública, v. 45, n. 1, p. 59-68, fev. 2011.

\section{Nota}

${ }^{1}$ A. M. Cervato-Mancuso participou da concepção, projeto, redação e aprovou a versão final do artigo. K. R. R. Vincha e D. A. Santiago participaram da análise e interpretação dos dados, redação e aprovação da versão final do artigo. 
Food and Nutrition Education as intervention practice: reflection and possible strengthening

Introduction: Nutritional interventions are historically configured by food distribution and expansion of knowledge, keeping current practices. To expand the field, the Marco de Referência de Educação Alimentar e Nutricional [Reference Landmark of Food and Nutrition Education] was set in 2012. Objective: Analyze the Brazilian scientific production about evaluation of interventions in Food and Nutrition Education prior to the landmark establishment. Methodology: Systematic research including articles published from 2000 to 2012. After a critical reading, the studies were divided, selected and classified by region, context and scenario. Results: 28 articles were selected and divided into three categories: institutional and study characteristics; intervention contexts and social actors involved; and organization and theoretical basis. It was detected that dietitians and the theme "nutrition" are prevalent in the health context. In the school setting, in the territory and in the work setting, the health promotion is approached by multi-professional teams. The objectives and evaluations of the interventions are related predominantly with numerical indicators, however, the territory studies approximate the landmark references by appropriating qualitative indicators. There was a succinct description of the theoretical and methodological bases, limiting the reproduction of the studies. Considerations: We point out the need for more intervention studies and its improvement by using the landmark as reference.

Key words: Food and Nutrition Education; intervention studies; review. 